\title{
Asymptotic distribution of entry times in a cellular automaton with annihilating particles
}

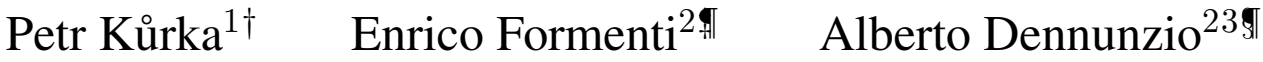 \\ ${ }^{1}$ Center for Theoretical Study, Academy of Sciences and Charles University in Prague, Czechia \\ ${ }^{2}$ Laboratoire I3S, Université Nice Sophia Antipolis, France \\ ${ }^{3}$ Dipartimento di Informatica, Sistemistica e Comunicazione, Università degli Studi di Milano-Bicocca, Italy
}

This work considers a cellular automaton (CA) with two particles: a stationary particle 1 and left-going one $\overline{1}$. When a $\overline{1}$ encounters a 1, both particles annihilate. We derive asymptotic distribution of appearence of particles at a given site when the CA is initialized with the Bernoulli measure with the probabilities of both particles equal to $1 / 2$.

Keywords: Cellular Automata, Particle Systems, Entry Times, Return Times

\section{Introduction}

Cellular automata are a simple formal model for complex systems. They consist of an infinite number of identical finite automata arranged over a regular lattice (here $\mathbb{Z}$ ). Each automaton updates its state according to its own state and the one of a fixed set of neighboring automata according to a local rule. All updates are synchronous.

The simplicity of the model contrasts with the great variety of different dynamical behaviors. Indeed, exactly this rich variety of behaviors and the ease of being simulated on computers made CA fortune. Actually, they are used in almost all scientific disciplines ranging from Mathematics to Computer Science and Natural Sciences. In particular, in Biology, Physics and Economics, they can be used as a discrete counterpart (in the sense of time) of interacting particle systems (IPS).

The advantage of modeling IPS by CA is that one can have information not only about limit distributions and particle densities but also on their spatial distribution.

On the other hand, as we have already mentioned, the dynamical behavior of CA is complex and not fully understood and IPS can help to understand the dynamics of some CA whenever it can be described in terms of particles or signals that move in a neutral background and interact on encounters. The general concept of a signal or particle (in the context of CA) has been elaborated in Formenti and Kürka (2007).

\footnotetext{
${ }^{\dagger}$ Email: kurka@cts.cuni.cz. This research was supported by the Research Program CTS MSM 0021620845

‡Email: enrico.formenti@unice.fr

§Email: dennunzioddisco.unimib.it

"Supported by the French National Research Agency project EMC (ANR-09-BLAN-0164) 
The simplest kind of particles interaction is the annihilation. The classical example is "Just gliders" studied in Gilman (1987). This system consists of a left-going particle $\overline{1}$ and a right-going particle 1 which annihilate on encounters. If the system starts in a Bernoulli measure with equal probabilities of both particles, then at a specified site both kinds of particles keep appearing with probability one, although their appearance is more and more rare as it has been shown by Kůrka and Maass (2002). Other peculiar particle systems and related CA models have been studied, see for example Fisch (1990).

In the present paper we address the question of how the time of appearance of a particle depends on the age of the system. We work with a simpler system called asymmetric gliders consisting of one stationary and one left-going particles annihilating on encounters. We show that the appearance of leftgoing particles time scales linearly with the age of the system and we derive the limit scaled distribution.

The paper is organized as follows. Section 2 and 3 introduce the symmetric and asymmetric gliders CA, respectively. Results are in Section 3 . Since the proofs of the main results require several technical lemmata and specific notations, we grouped them in Section 4 . The final section draws some conclusions and give some ideas for future work.

\section{Symmetric gliders}

Let $A$ be a finite alphabet. A $I D C A$ configuration is a function from $\mathbb{Z}$ to $A$. The $1 D C A$ configuration set $A^{\mathbb{Z}}$ is usually equipped with the metric $d$ defined as follows

$$
\forall c, c^{\prime} \in A^{\mathbb{Z}}, d\left(c, c^{\prime}\right)=2^{-n} \text {, where } n=\min \left\{i \geq 0: c_{i} \neq c_{i}^{\prime} \text { or } c_{-i}^{\prime} \neq c_{-i}^{\prime}\right\} .
$$

If $A$ is finite, $A^{\mathbb{Z}}$ is a compact, totally disconnected and perfect topological space (i.e., $A^{\mathbb{Z}}$ is a Cantor space). For any pair $i, j \in \mathbb{Z}$, with $i \leq j$, and any configuration $x \in A^{\mathbb{Z}}$ we denote by $x_{[i, j]}$ the word $x_{i} \cdots x_{j} \in A^{j-i+1}$, i.e., the portion of $c$ inside the interval $[i, j]$. In the previous notation, $[i, j]$ can be replaced by $[i, j)$ with the obvious meaning. A cylinder of block $u \in A^{k}$ and position $i \in \mathbb{Z}$ is the set $[u]_{i}=\left\{x \in A^{\mathbb{Z}}: x_{[i, i+k-1]}=u\right\}$. Cylinders are clopen sets w.r.t. the metric $d$ and they form a basis for the topology induced by $d$.

A $I D C A$ is a structure $\langle 1, A, r, f\rangle$, where $A$ is the alphabet, $r \in \mathbb{N}$ is the radius and $f: A^{2 r+1} \rightarrow A$ is the local rule of the automaton. The local rule $f$ induces a global rule $F: A^{\mathbb{Z}} \rightarrow A^{\mathbb{Z}}$ defined as follows,

$$
\forall c \in A^{\mathbb{Z}}, \forall i \in \mathbb{Z}, \quad F(c)_{i}=f\left(c_{i-r}, \ldots, c_{i}, \ldots, c_{i+r}\right) .
$$

In Gilman (1987), Gilman introduced a CA called Just Gliders (or Symmetric Gliders) which is formally defined as $\langle 1,\{\overline{1}, 0,1\}, 1, g\rangle$ where $g:\{\overline{1}, 0,1\}^{3} \rightarrow\{\overline{1}, 0,1\}$ is such that

$$
\forall(x, y, z) \in\{\overline{1}, 0,1\}^{3}, \quad g(x, y, z)= \begin{cases}1 & \text { if } x=1, y \geq 0 \text { and } y+z \geq 0 \\ \overline{1} & \text { if } z=\overline{1}, y \leq 0 \text { and } x+y \leq 0 \\ 0 & \text { otherwise }\end{cases}
$$

In this context a symbol 1 (resp. $\overline{1}$ ) is interpreted as a right-going (resp. left-going) particle and 0 is the neutral background. Figure 1 shows an example of evolution of Just Gliders from a random initial configuration. 


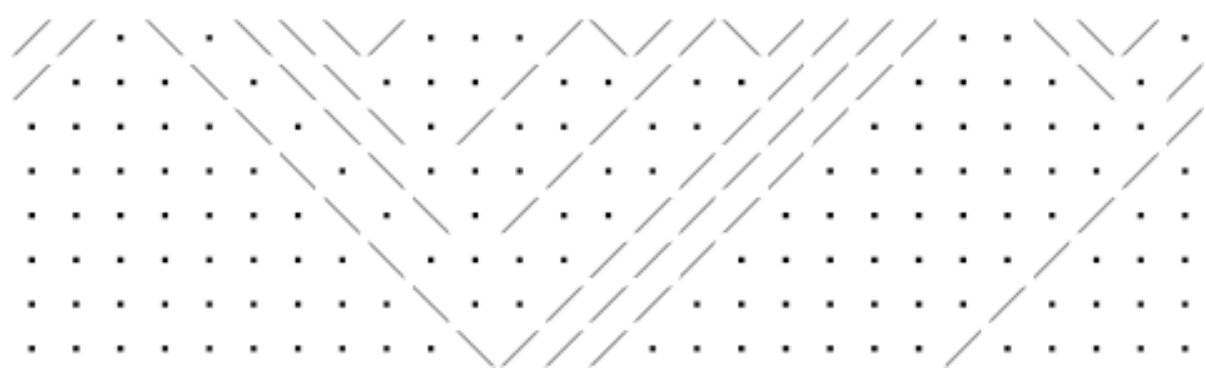

Fig. 1: Symmetric gliders.

Consider a Bernoulli measure on $\{\overline{1}, 0,1\}^{\mathbb{Z}}$, i.e., a sequence of independent identically distributed random variables $X=\left(X_{i}\right)_{i \in \mathbb{Z}}$ over $\{\overline{1}, 0,1\}$ such that $\forall i \in \mathbb{Z}, \mathbb{P}\left[X_{i}=\overline{1}\right]=\mathbb{P}\left[X_{i}=1\right]=p, \mathbb{P}\left[X_{i}=\right.$ $0]=1-2 p=q$. Then, for any $\mathrm{CA}$ global rule $F$ and any $n \in \mathbb{N}, F^{n}(X)_{0}$ is also a random variable whose distribution depends on the initial distribution of $X$.

Definition 1 (Entry time) For $a \in\{\overline{1}, 0,1\}$, the entry time into $[a]_{0}$ (appearance of a particle a) after time $n$ at position 0 is

$$
T_{n}^{a}(X)=\min \left\{k \geq 0: F^{n+k}(X)_{0}=a\right\} .
$$

Since $F$ commutes with $\sigma$, the entry times at any position $s \in \mathbb{Z}$ have the same distribution as $T_{n}^{a}$. In Gilman (1987), the following result has been proven.

Theorem 1 Let $F$ be the global function of Just Gliders $C A$. If $\mathbb{P}\left[X_{i}=1\right]>\mathbb{P}\left[X_{i}=\overline{1}\right]$ then

$$
\mathbb{P}\left[\forall n \in \mathbb{N}, \exists k \in \mathbb{N} \text { s.t. } F^{n+k}(X)_{0}=\overline{1}\right]=\mathbb{P}\left[\forall n \in \mathbb{N}, T_{n}^{\overline{1}}(X)<\infty\right]=0
$$

Then Kůrka and Maass (2002) proved the following

Theorem 2 Let $F$ be the global function of Just Gliders $C A$. If $\mathbb{P}\left[X_{i}=1\right]=\mathbb{P}\left[X_{i}=\overline{1}\right]$ then

1. $\mathbb{P}\left[\forall n \in \mathbb{N}, \exists k \in \mathbb{N}\right.$ s.t. $\left.F^{n+k}(X)_{0} \neq 0\right]=1$;

2. $\mathbb{P}\left[\forall n \in \mathbb{N}, T_{n}^{a}(X)<\infty\right]=1$ for $a \in\{\overline{1}, 1\}$;

3. $\forall n \in \mathbb{N}, \mathbb{P}\left[T_{n}^{a}(X)<\infty\right]=1$ for $a \in\{\overline{1}, 1\}$;

4. $\lim _{n \rightarrow \infty} \mathbb{P}\left[F^{n}(X)_{0}=0\right]=1$.

\section{Asymmetric gliders}

In this paper we consider a similar CA that we call Asymmetric Gliders, $\langle 1,\{\overline{1}, 0,1\}, 1, f\rangle$ and $f$ : $\{\overline{1}, 0,1\}^{3} \rightarrow\{\overline{1}, 0,1\}$ is defined as follows

$$
\forall(x, y, z) \in\{\overline{1}, 0,1\}^{3}, \quad f(x, y, z)= \begin{cases}1 & \text { if } y=1 \text { and } z \neq \overline{1} \\ \overline{1} & \text { if } y \neq 1 \text { and } z=\overline{1} \\ 0 & \text { otherwise }\end{cases}
$$




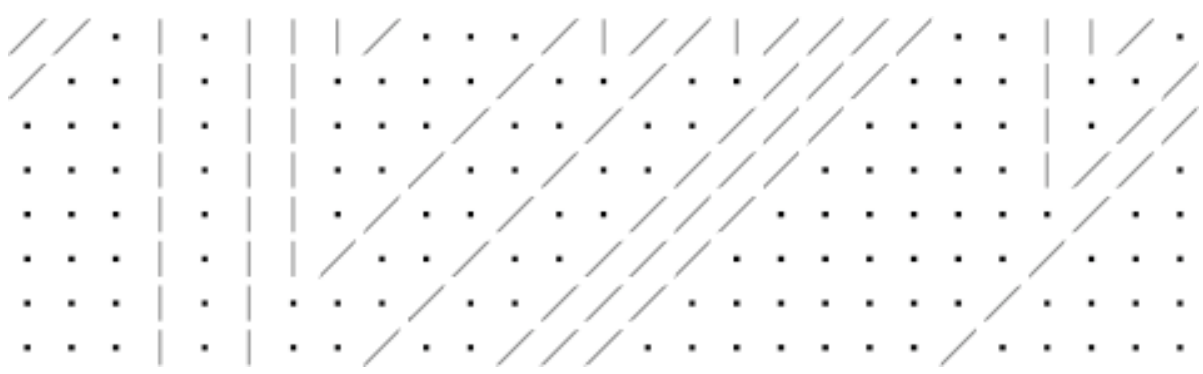

Fig. 2: Asymmetric gliders.

The symbol 1 can be interpreted as a stationary particle, $\overline{1}$ is a left-going particle and 0 is the neutral background. It is clear from the definition of $f$ that a particle 1 and a $\overline{1}$ annihilate when they meet (Figure 2. In the sequel, the symbols of $A$ are weighted naturally, namely, 0 with 0,1 with 1 and $\overline{1}$ with -1 . Thus, for example, $1+\overline{1}=0$ and $\overline{1}+\overline{1}=-2$ and so on.

Again, we consider a Bernoulli measure on $\{\overline{1}, 0,1\}^{\mathbb{Z}}$, i.e., a sequence of independent identically distributed random variables $X=\left(X_{i}\right)_{i \in \mathbb{Z}}$ over $\{\overline{1}, 0,1\}$ such that $\forall i \in \mathbb{Z}, \mathbb{P}\left[X_{i}=\overline{1}\right]=\mathbb{P}\left[X_{i}=1\right]=p$, $\mathbb{P}\left[X_{i}=0\right]=1-2 p=q$. Then, for any CA global rule $F$ and any $n \in \mathbb{N}, F^{n}(X)_{0}$ is also a random variable whose distribution depends on the initial distribution of $X$.

Proposition 1 Let $F$ be the global function of Asymmetric Gliders CA. If $\forall i \in \mathbb{Z}, \mathbb{P}\left[X_{i}=1\right]=\mathbb{P}\left[X_{i}=\right.$ $\overline{1}] \leq 1 / 2$ then

1. $\lim _{n \rightarrow \infty} \mathbb{P}\left[F^{n}(X)_{0}=0\right]=1$;

2. $\lim _{n \rightarrow \infty} \mathbb{P}\left[T_{n}^{0}(X)=0\right]=1$;

3. $\lim _{n \rightarrow \infty} \mathbb{P}\left[T_{n}^{1}(X)=\infty\right]=1$.

Proof: The following relations between $F^{n}(X)_{0}$ and the random variables $X_{i}$ hold

$$
\begin{aligned}
& F^{n}(X)_{0}=1 \quad \Leftrightarrow \quad \forall k \leq n, \sum_{i=0}^{k} X_{i}>0 \\
& F^{n}(X)_{0}=\overline{1} \quad \Leftrightarrow \quad \forall k \leq n, \sum_{i=k}^{n} X_{i}<0 \\
& F^{n}(X)_{0}=0 \quad \Leftrightarrow \quad \exists k \leq n, \sum_{i=0}^{k} X_{i} \leq 0 \text { and } \exists k \leq n, \sum_{i=k}^{n} X_{i} \geq 0
\end{aligned}
$$

Since $\sum_{i=0}^{n} X_{i}$ is a recurrent Markov chain, we get

$$
\lim _{n \rightarrow \infty} \mathbb{P}\left[F^{n}(X)_{0}=1\right]=\lim _{n \rightarrow \infty} \mathbb{P}\left[F^{n}(X)_{0}=\overline{1}\right]=0
$$


Therefore, it follows that $\lim _{n \rightarrow \infty} \mathbb{P}\left[F^{n}(X)_{0}=0\right]=1, \lim _{n \rightarrow \infty} \mathbb{P}\left[T_{n}^{0}(X)=0\right]=1$, and also $\lim _{n \rightarrow \infty} \mathbb{P}\left[T_{n}^{1}(X)=\infty\right]=1$.

As a consequence of Proposition 1 as $n \rightarrow \infty, T_{n}^{0}(X) \rightarrow 0$ and $T_{n}^{1}(X) \rightarrow \infty$ in probability. Moreover, since for any $n \in \mathbb{N}$ the set of events such that $T_{n}^{1}(X)=\infty$ is contained in the one such that $\lim _{n \rightarrow \infty} T_{n}^{1}(X)=\infty$, it holds that $\mathbb{P}\left[\lim _{n \rightarrow \infty} T_{n}^{1}(X)=\infty\right] \geq \lim _{n \rightarrow \infty} \mathbb{P}\left[T_{n}^{1}(X)=\infty\right]=1$, and, hence, $T_{n}^{1}(X) \rightarrow \infty$ almost surely.

Proposition 2 Let $F$ be the global function of Asymmetric Gliders $C A$. If $\forall i \in \mathbb{Z}, \mathbb{P}\left[X_{i}=1\right]=\mathbb{P}\left[X_{i}=\right.$ $\overline{1}]=1 / 2$ then

1. $\forall x, \lim _{n \rightarrow \infty} \mathbb{P}\left[T_{n}^{\overline{1}}(X)>x\right]=1 ;$

2. $\forall n \in \mathbb{N}, \mathbb{E}\left(T_{n}^{\overline{1}}\right)=\infty$.

Theorem 3 Let $F$ be the global function of Asymmetric Gliders $C A$. If $\forall i \in \mathbb{Z}, \mathbb{P}\left[X_{i}=1\right]=\mathbb{P}\left[X_{i}=\right.$ $\overline{1}]=1 / 2$ then

$$
\lim _{n \rightarrow \infty} \mathbb{P}\left[\frac{T_{n}^{\overline{1}}(X)}{n} \leq x\right]=\frac{2}{\pi} \arctan \sqrt{x} .
$$

In the general case with $p \leq 1 / 2$ we have $\operatorname{Var}(X)=2 p$ so the time scales by $\sqrt{2 p}$. Hence, we can give the following.

Conjecture 1 Let $F$ be the global function of Asymmetric Gliders $C A$. If $\forall i \in \mathbb{Z}, \mathbb{P}\left[X_{i}=1\right]=\mathbb{P}\left[X_{i}=\right.$ $\overline{1}] \leq 1 / 2$ then

$$
\lim _{n \rightarrow \infty} \mathbb{P}\left[\frac{T_{n}^{\overline{1}}(X)}{n} \leq x\right]=\frac{2}{\pi} \arctan \sqrt{2 p x}
$$

\section{Proof of main results}

Notation. For the sake of simplicity, from now on $T_{n}^{\overline{1}}$ is denoted $T_{n}$ whenever no misunderstanding is possible.

First of all, we should precise the definition of what we mean by annihilation of particles 1 and $\overline{1}$.

Definition 2 (Annihilation) A particle 1 at the position $n$ is annihilated with the particle $\overline{1}$ at position $n+k$, if $F^{k-1}(X)_{n}=1$ and $F^{k-1}(X)_{n+1}=\overline{1}$.

Denote by $Y_{n}$ the number of particles 1 in the interval $[0, n)$ which are not annihilated with any particle $\overline{1}$ in the interval $[0, n)$. Then, $Y_{0}=0$ and $Y_{n+1}=\max \left\{0, Y_{n}+X_{n}\right\}$, so $Y$ is a Markov chain whose transition probabilities are in Figure 3 .

For the probabilities $P_{n, m}=\mathbb{P}\left[Y_{n}=m\right]$ we have $P_{0,0}=1$ and $P_{n, m}=0$ for $m>n$. The balance equations for the Markov chain $Y$ give

$$
\begin{aligned}
P_{n+1,0} & =(1-p) \cdot P_{n, 0}+p \cdot P_{n, 1} \\
P_{n+1, m} & =p \cdot P_{n, m-1}+(1-2 p) \cdot P_{n, m}+p \cdot P_{n, m+1} \text { for } m>0
\end{aligned}
$$




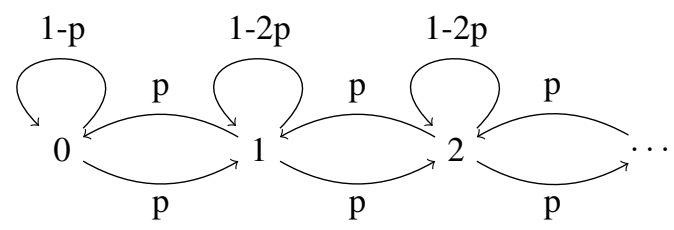

Fig. 3: The Markov chain $Y$ above defined.

For a fixed $n \geq 0$ consider the stochastic process $Z$ such that $Z_{0}=Y_{n}$ and $Z_{m+1}=Z_{m}+X_{n+m}$. If $Z_{0}, \ldots Z_{m}$ are all nonnegative, then $Z_{m}$ is the number of particles 1 in $[0, n+m)$ which are not annihilated with any particle $\overline{1}$ in $[0, n+m)$. For $m \geq l$ define the entry times for $Z$ as follows

$$
S_{m, l}=\min \left\{t>0: Z_{t}=l \mid Z_{0}=m\right\}
$$

and the associated probabilities

$$
Q_{m, k}=\mathbb{P}\left[S_{m+l, l}=k\right]=\mathbb{P}\left[S_{m, 0}=k\right] .
$$

Remark that for $m_{2}>m_{1}>m_{0}$ we have $S_{m_{2}, m_{0}}=S_{m_{2}, m_{1}}+S_{m_{1}, m_{0}}$, so $S_{m, 0}$ is the sum of $m$ independent random variables which have all the same distribution as $S_{1,0}$. Thus $Q_{0,1}=q, Q_{0,2}=2 p^{2}$, $Q_{1,1}=p, Q_{m, 1}=0$ for $m>1, Q_{1,2}=p(1-2 p)=p q, Q_{2,2}=p^{2}$ and $Q_{m, k}=0$ for $m>k$. According to the equilibrium equation of the Markov chain $Z$ one finds

$$
\begin{aligned}
Q_{0, k+1} & =2 p \cdot Q_{1, k} \text { for } m=0 \\
Q_{1, k+1} & =q \cdot Q_{1, k}+p \cdot Q_{2, k} \text { for } m=1 \\
Q_{m, k+1} & =p \cdot Q_{m-1, k}+q \cdot Q_{m, k}+p \cdot Q_{m+1, k} \text { for } m>1
\end{aligned}
$$

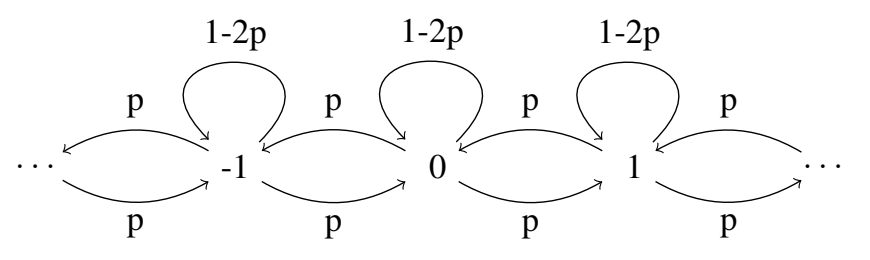

Fig. 4: The Markov chain $Z$ above introduced.

Remark that $T_{n}=k$ iff $Z_{k+1}=-1$ and $Z_{j} \geq 0$ for all $j \leq k$. Thus, if $Z_{0}=m$, then $T_{n}=k$ iff $S_{m,-1}=k+1$. So, for the entry time $T_{n}$ and the related probabilities $\mathbb{P}\left[T_{n}=k \mid Z_{0}=m\right]$ and $R_{n, k}:=\mathbb{P}\left[T_{n}=k\right]$, we have

$$
\begin{aligned}
T_{n} & =\chi_{\left[Y_{n}=m\right]} \cdot S_{m,-1} \\
\mathbb{P}\left[T_{n}=k \mid Z_{0}=m\right] & =\mathbb{P}\left[S_{m,-1}=k+1\right]=Q_{m+1, k+1} \\
R_{n, k} & =\mathbb{P}\left[T_{n}=k\right]=\sum_{m=0}^{\min (n, k+1)} P_{n, m} \cdot Q_{m+1, k+1}
\end{aligned}
$$


When $\forall i \in \mathbb{Z}, \mathbb{P}\left[X_{i}=1\right]=\mathbb{P}\left[X_{i}=\overline{1}\right]=1 / 2$ and $\mathbb{P}\left[X_{i}=0\right]=0$ from the definitions of the probabilities $P$ and $Q$ we obtain the following matrices.

$$
P=\begin{array}{c|cccccc} 
& 0 & 1 & 2 & 3 & 4 & \ldots \\
\hline 0 & 1 & 0 & 0 & 0 & 0 & \cdots \\
1 & \frac{1}{2} & \frac{1}{2} & 0 & 0 & 0 & \cdots \\
2 & \frac{2}{4} & \frac{1}{4} & \frac{1}{4} & 0 & 0 & \cdots \\
3 & \frac{3}{8} & \frac{3}{8} & \frac{1}{8} & \frac{1}{8} & 0 & \ldots \\
4 & \frac{6}{16} & \frac{4}{16} & \frac{4}{16} & \frac{1}{16} & \frac{1}{16} & \cdots \\
\vdots & \vdots & \vdots & \vdots & \vdots & \vdots & \ddots
\end{array}
$$

$Q=$\begin{tabular}{c|cccccccc} 
& 1 & 2 & 3 & 4 & 5 & 6 & 7 & $\ldots$ \\
\hline 0 & 0 & $\frac{1}{2}$ & 0 & $\frac{1}{8}$ & 0 & $\frac{1}{16}$ & 0 & $\cdots$ \\
1 & $\frac{1}{2}$ & 0 & $\frac{1}{8}$ & 0 & $\frac{1}{16}$ & 0 & $\frac{5}{128}$ & $\ldots$ \\
2 & 0 & $\frac{1}{4}$ & 0 & $\frac{1}{8}$ & 0 & $\frac{5}{64}$ & 0 & $\ldots$ \\
3 & 0 & 0 & $\frac{1}{8}$ & 0 & $\frac{3}{32}$ & 0 & $\frac{9}{128}$ & $\ldots$ \\
4 & 0 & 0 & 0 & $\frac{1}{16}$ & 0 & $\frac{1}{16}$ & 0 & $\ldots$ \\
$\vdots$ & $\vdots$ & $\vdots$ & $\vdots$ & $\vdots$ & $\vdots$ & $\vdots$ & $\vdots$ & $\ddots$
\end{tabular}

Next lemmata will give closed formulas for $P$ and $Q$.

Lemma 1 If $\forall i \in \mathbb{Z}, \mathbb{P}\left[X_{i}=1\right]=\mathbb{P}\left[X_{i}=\overline{1}\right]=1 / 2$ then for all $n, m \in \mathbb{N}$ with $m \leq n$, the following equalities hold on the probabilities $P_{n, m}$ :

$$
\begin{aligned}
P_{2 n, 2 m+2}=P_{2 n, 2 m+1} & =\left(\begin{array}{c}
2 n \\
n+m+1
\end{array}\right) \cdot 2^{-2 n} \\
P_{2 n+1,2 m}=P_{2 n+1,2 m+1} & =\left(\begin{array}{c}
2 n+1 \\
n+m+1
\end{array}\right) \cdot 2^{-2 n-1} \\
P_{n, m} & =\left(\begin{array}{c}
n \\
\left\lceil\frac{n+m}{2}\right\rceil
\end{array}\right) \cdot 2^{-n}
\end{aligned}
$$

Proof: We have $P_{0,0}=1$. By induction, assume that equalities (6) and (7) are true for some value $n$ and for all $m \leq n$. Since from equations (1) and 22, $P_{n+1,0}=\left(P_{n, 0}+P_{n, 1}\right) / 2$ and $P_{n+1, m}=$ $\left(P_{n, m-1}+P_{n, m+1}\right) / 2$, it follows that (6) and (7) are true for $n+1$ and for all $m \leq n+1$. Thus, (6) and (7) hold, and, as a consequence, equality (8) too.

According to Rényi (1970), the following relation holds for the $Q_{m, k}$

$$
Q_{1,2 k-1}=Q_{0,2 k}=\frac{1}{k \cdot 2^{2 k-1}}\left(\begin{array}{c}
2 k-2 \\
k-1
\end{array}\right)=(-1)^{k-1} \cdot\left(\begin{array}{c}
1 / 2 \\
k
\end{array}\right)
$$

Lemma 2 If $\forall i \in \mathbb{Z}, \mathbb{P}\left[X_{i}=1\right]=\mathbb{P}\left[X_{i}=\overline{1}\right]=1 / 2$ then for all $m$ with $0<m \leq k$ the following equalities on the quantities $Q_{m, k}$ hold.

$$
Q_{m, k}=0, \quad \text { if } \bmod _{2}(k+m)=1
$$

while

$$
\begin{aligned}
Q_{2 m, 2 k} & =\frac{m}{k}\left(\begin{array}{c}
2 k \\
k-m
\end{array}\right) \cdot 2^{-2 k} \\
Q_{2 m+1,2 k+1} & =\frac{2 m+1}{2 k+1}\left(\begin{array}{c}
2 k+1 \\
k-m
\end{array}\right) \cdot 2^{-2 k-1} \\
Q_{m, k} & =\frac{m}{k}\left(\begin{array}{c}
k \\
(k-m) / 2
\end{array}\right) \cdot 2^{-k},
\end{aligned}
$$

if $\bmod _{2}(k+m)=0$, where $\bmod _{2}(m)$ is $m \bmod 2$. 
Proof: Since $q=0$, if $\bmod _{2}(k+m)=1$ Equality (10) follows from the definition of $Q_{m, k}$. Using that $Q_{2, k}=2 \cdot Q_{1, k+1}, Q_{m+1, k}=2 \cdot Q_{m, k+1}-Q_{m-1, k}$ (for $m \geq 1$ ), and Equation (9), Equalities from (11) to (13), are true for $Q_{1, k}$ and $Q_{2, k}$. The thesis is obtained by proceeding by finite induction on $m$.

Using the expressions found in Lemmata 1 and 2 and substituting them in the definition of $R_{n, k}=$ $\mathbb{P}\left[T_{n}=k\right]$, one can easily find the following.

$$
\begin{aligned}
R_{2 n, 2 k} & =2^{-2 n-2 k-1} \sum_{m=0}^{\min (n, k)}\left(\begin{array}{c}
2 n \\
n+m
\end{array}\right)\left(\begin{array}{c}
2 k+1 \\
k-m
\end{array}\right) \frac{2 m+1}{2 k+1} \\
R_{2 n, 2 k+1} & =2^{-2 n-2 k-2} \sum_{m=0}^{\min (n, k)}\left(\begin{array}{c}
2 n \\
n+m+1
\end{array}\right)\left(\begin{array}{c}
2 k+2 \\
k-m
\end{array}\right) \frac{m+1}{k+1} \\
R_{2 n+1,2 k} & =2^{-2 n-2 k-2} \sum_{m=0}^{\min (n, k)}\left(\begin{array}{c}
2 n+1 \\
n+m+1
\end{array}\right)\left(\begin{array}{c}
2 k+1 \\
k-m
\end{array}\right) \frac{2 m+1}{2 k+1} \\
R_{2 n+1,2 k+1} & =2^{-2 n-2 k-3} \sum_{m=0}^{\min (n, k)}\left(\begin{array}{c}
2 n+1 \\
n+m+1
\end{array}\right)\left(\begin{array}{c}
2 k+2 \\
k-m
\end{array}\right) \frac{m+1}{k+1}
\end{aligned}
$$

which can be summed up as follows

$$
R_{n, k}=2^{-n-k-1} \sum_{m=0}^{\min \left(\left\lfloor\frac{n}{2}\right\rfloor,\left\lfloor\frac{k}{2}\right\rfloor\right)}\left(\begin{array}{c}
n \\
\left\lfloor\frac{n}{2}\right\rfloor+m+\ell_{n, k}
\end{array}\right)\left(\begin{array}{c}
k+1 \\
\left\lfloor\frac{k}{2}\right\rfloor-m
\end{array}\right) \frac{2 m+1+\bmod _{2}(k)}{k+1}
$$

where $\ell_{n, k}=\max \left\{\bmod _{2}(n), \bmod _{2}(k)\right\}$.

Finally, we will use the following approximation formula of binomial distribution by the normal distribution.

Theorem 4 (Rényi (1970)) Let $k_{n}$ be a sequence of positive integers such that $\left|2 k_{n}-n\right|<a \sqrt{n}$ for some constant $a$. Then

$$
\left(\begin{array}{c}
n \\
k_{n}
\end{array}\right)=\frac{2^{n+1} \cdot e^{-\left(2 k_{n}-n\right)^{2} / 2 n}}{\sqrt{2 \pi n}}(1+\mathcal{O}(1 / n))
$$

and the constant in the remainder $\mathcal{O}(1 / n)$ depends only on a.

\section{Proof of Proposition 2:}

1. Using the approximation given by Theorem 4 one finds

$$
\lim _{n \rightarrow \infty} P_{n, m}=\lim _{n \rightarrow \infty} \frac{2 e^{-m^{2} / 2 n}}{\sqrt{2 \pi n}}=0
$$

and hence

$$
\lim _{n \rightarrow \infty} \mathbb{P}\left[T_{n} \leq \ell\right]=\lim _{n \rightarrow \infty} \sum_{k=0}^{\ell} \sum_{m=0}^{\min (n, k)} P_{n, m} \cdot Q_{m+1, k+1}=0
$$


2. To prove $\mathbb{E}\left(T_{n}\right)=\infty$ we prove first that $\mathbb{E}\left(S_{m, 1}\right)=\infty$ for each $m$. Again, using Theorem 4 , one finds

$$
Q_{m, k}=\frac{m}{k} \cdot\left(\begin{array}{c}
k \\
\left\lceil\frac{k-m}{2}\right\rceil
\end{array}\right) \cdot 2^{-k} \approx \frac{m}{k} \cdot 2^{k+1} \frac{e^{-\frac{m^{2}}{2 k}}}{\sqrt{2 \pi k}} \cdot 2^{-k}=\frac{2 m \cdot e^{-\frac{m^{2}}{2 k}}}{k \sqrt{2 \pi k}}
$$

whenever $m<\sqrt{k}$ and $\bmod _{2}(k+m)=0$. For each $\ell$ we then have

$$
\mathbb{E}\left(S_{m, 0}\right)=\sum_{k=1}^{\infty} k \cdot Q_{m, k} \geq \sum_{k=\ell}^{\infty} \frac{2 m \cdot e^{-\frac{m^{2}}{2 k}}}{\sqrt{2 \pi k}}=\infty .
$$

Since $\mathbb{E}\left(T_{n}\right)$ is a finite linear combination of $\mathbb{E}\left(S_{0,-1}\right), \ldots, \mathbb{E}\left(S_{n,-1}\right)$, we get $\mathbb{E}\left(T_{n}\right)=\infty$ as well.

Proof of Theorem 3: Recall that the characteristic function of a discrete distribution with $\mathbb{P}[X=n]=p_{n}$ is $\varphi(t)=\sum_{n=0}^{\infty} p_{n} e^{i n t}$, where $i$ is the imaginary unit. Since by Lemma $2 Q_{m, k}=0$ if $\bmod _{2}(k+m)=1$, for the characteristic function $\varphi$ of $S_{1,0}$ we obtain

$$
\varphi(t)=Q_{1,1} e^{i t}+Q_{1,3} e^{3 i t}+Q_{1,5} e^{5 i t}+\cdots
$$

which, by using Equation 99, turns into

$$
\begin{aligned}
\varphi(t) & =\left(\begin{array}{c}
1 / 2 \\
1
\end{array}\right) e^{i t}-\left(\begin{array}{c}
1 / 2 \\
2
\end{array}\right) e^{3 i t}+\left(\begin{array}{c}
1 / 2 \\
3
\end{array}\right) e^{5 i t}-\cdots \\
& =e^{-i t}\left[1-\left(1-\left(\begin{array}{c}
1 / 2 \\
1
\end{array}\right) e^{2 i t}+\left(\begin{array}{c}
1 / 2 \\
2
\end{array}\right) e^{4 i t}-\cdots\right)\right] \\
& =e^{-i t}\left(1-\sqrt{1-e^{2 i t}}\right)
\end{aligned}
$$

Thus, finally we get

$$
\lim _{n \rightarrow \infty} \varphi\left(\frac{t}{n^{2}}\right)^{n}=\lim _{n \rightarrow \infty}\left(1-\sqrt{1-e^{2 i t / n^{2}}}\right)^{n}=\lim _{n \rightarrow \infty}\left(1-\frac{\sqrt{-2 i t}}{n}\right)^{n}=e^{-\sqrt{-2 i t}}
$$

which is the characteristic function of a random variable with distribution function

$$
G(x)=2(1-\Phi(1 / \sqrt{x}))
$$

and density

$$
g(x)=G^{\prime}(x)=\frac{1}{\sqrt{2 \pi x^{3}}} \cdot e^{-1 / 2 x},
$$

where $\Phi(x)$ is the normal distribution function. Figure 5 plots both $G(x)$ and $g(x)$.

Recall that $\mathbb{E}\left(S_{m, 0}\right)=\infty$ (see Equation 15 . Denote by $G_{m}(x)=\mathbb{P}\left[S_{m, 0} \leq x\right]$ the distribution function of $S_{m, 0}$. Since the characteristic function of $S_{m, 0}$ is $\varphi_{m}(t)=\varphi(t)^{m}$, we get

$$
\lim _{m \rightarrow \infty} G_{m+1}\left(m^{2} x\right)=\lim _{m \rightarrow \infty} \mathbb{P}\left[\frac{S_{m,-1}}{m^{2}}<x\right]=G(x)=2(1-\Phi(1 / \sqrt{x}))
$$




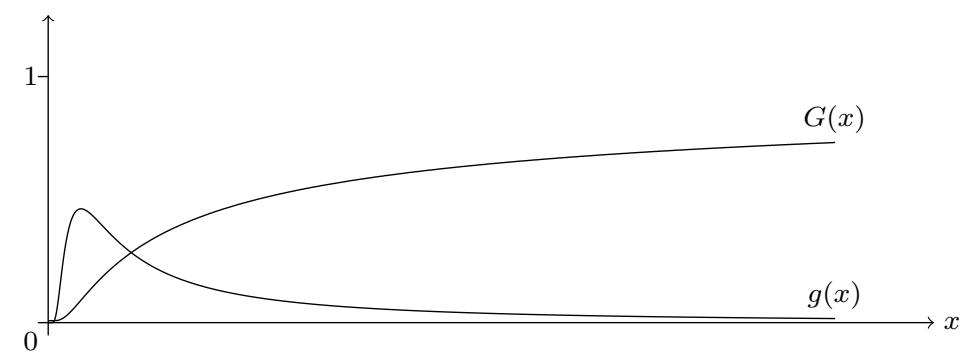

Fig. 5: Asymptotic distribution functions and densities of $\frac{S_{m, 0}}{m^{2}}$.

Denote by $H_{n}(x)=\mathbb{P}\left[T_{n} \leq x\right]$ the distribution function of $T_{n}$. We estimate $H_{n}(n x)=\mathbb{P}\left[T_{n} / n \leq x\right]$. For a fixed $x>0$ and $0<a<b$ we have

$$
\begin{aligned}
& A_{n}(a):=\sum_{m=0}^{\lfloor a \sqrt{n}\rfloor} P_{n, m} \cdot G_{m+1}(n x) \leq a \sqrt{n} \cdot P_{n, 0} \leq \frac{2 a}{\sqrt{2 \pi}}(1+\mathcal{O}(1 / \sqrt{n})) \\
& B_{n}(b):=\sum_{m=\lceil b \sqrt{n}\rceil}^{n} P_{n, m} \cdot G_{m+1}(n x) \leq G_{\lceil b \sqrt{n}\rceil}(n x)
\end{aligned}
$$

so

$$
\lim _{n \rightarrow \infty} A_{n}(a) \leq \frac{2 a}{\sqrt{2 \pi}} \text { and } \quad \lim _{n \rightarrow \infty} B_{n}(b) \leq G\left(x / b^{2}\right)
$$

We get

$$
\begin{aligned}
H_{n}(n x) & =\sum_{m=0}^{n} P_{n, m} \cdot G_{m+1}(n x) \\
& =A_{n}(a)+B_{n}(b)+\sum_{m=\lceil a \sqrt{n}\rceil}^{\lfloor b \sqrt{n}\rfloor} P_{n, m} \cdot G_{m+1}(n x) \\
& =A_{n}(a)+B_{n}(b)+\sum_{m=\lceil a \sqrt{n}\rceil}^{\lfloor b \sqrt{n}\rfloor} \frac{2 e^{-\left(m+\bmod _{2}(m+n)\right)^{2} / 2 n}}{\sqrt{2 \pi n}} \cdot(1+\mathcal{O}(1 / n)) \cdot G_{m+1}(n x)
\end{aligned}
$$

Some approximations functions $H_{n}(n x)$ are plotted in Figure 6 Remark how they quickly converge to $H(x)$. Using variable $y=m / \sqrt{n}$, we get $n x=x m^{2} / y^{2}$ and compute $H(x)=\lim _{n \rightarrow \infty} H_{n}(n x)$

$$
\begin{aligned}
H(x) & =\lim _{n \rightarrow \infty}\left(A_{n}(a)+B_{n}(b)+\sum_{m=\lceil a \sqrt{n}\rceil}^{\lfloor b \sqrt{n}\rfloor} \frac{2 e^{-y^{2} / 2}}{\sqrt{2 \pi n}} \cdot G_{m+1}\left(m^{2} x / y^{2}\right)\right) \\
& =\lim _{n \rightarrow \infty}\left(A_{n}(a)+B_{n}(b)\right)+\lim _{n \rightarrow \infty} \sum_{m=\lceil a \sqrt{n}\rceil}^{\lfloor b \sqrt{n}\rfloor} \frac{2 e^{-y^{2} / 2}}{\sqrt{2 \pi n}} \cdot G_{m+1}\left(m^{2} x / y^{2}\right)
\end{aligned}
$$




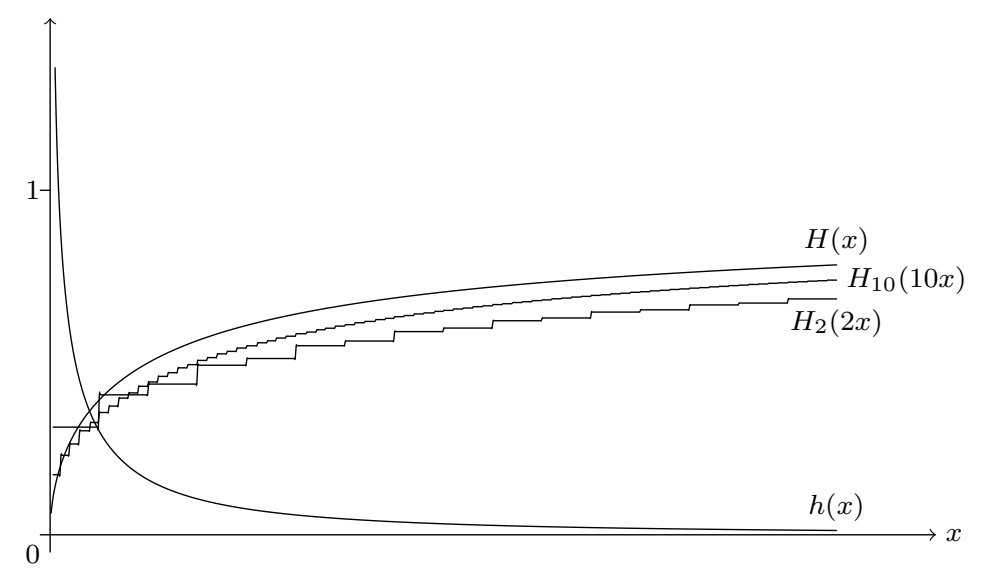

Fig. 6: Asymptotic distribution functions and densities of $\frac{T_{n}}{n}$.

and, by using 16 , we obtain (recall that $y=m / \sqrt{n} \in[a, b]$ )

$$
H(x)=\lim _{n \rightarrow \infty}\left(A_{n}(a)+B_{n}(b)\right)+\sqrt{\frac{2}{\pi}} \sum_{y=a}^{b} e^{-y^{2} / 2} \cdot G_{m+1}\left(m^{2} x / y^{2}\right)
$$

Since $\lim _{a \rightarrow 0} \lim _{n \rightarrow \infty} A_{n}(a)=0$ and $\lim _{b \rightarrow \infty} \lim _{n \rightarrow \infty} B_{n}(b)=0$ (see Inequalities in 17 , we have

$$
\begin{aligned}
H(x) & =\sqrt{\frac{2}{\pi}} \cdot \lim _{a \rightarrow 0} \lim _{b \rightarrow \infty} \sum_{y=a}^{b} e^{-y^{2} / 2} \cdot G_{m+1}\left(m^{2} x / y^{2}\right) \\
& =\sqrt{\frac{2}{\pi}} \int_{0}^{\infty} e^{-y^{2} / 2} \cdot G\left(x / y^{2}\right) d y
\end{aligned}
$$

which gives for the density

$$
\begin{aligned}
h(x)=H^{\prime}(x) & =\sqrt{\frac{2}{\pi}} \cdot \int_{0}^{\infty} e^{-y^{2} / 2} \cdot g\left(x / y^{2}\right) \cdot y^{-2} d y \\
& =\frac{1}{\pi \sqrt{x^{3}}} \int_{0}^{\infty} y \cdot e^{-\frac{y^{2}}{2}\left(1+\frac{1}{x}\right)} d y=\frac{1}{\pi \sqrt{x}(x+1)}
\end{aligned}
$$

and hence

$$
H(x)=\int h(x) d x=\frac{2}{\pi} \arctan \sqrt{x}
$$




\section{Conclusions}

In this paper we set up some formal tools that have help to study and to exactly derive the distribution of entry time for CA viewed a particle system consisting of a stationary particle and a left-going particle.

The program is to develop formal tools in order to be able to study more complex situations starting by the symmetric case for example. Another interesting case would consider particles with speed different from 1 or 0 allowing in this way more complex interactions between particles other than annihilation.

\section{References}

R. Fisch. The one-dimensional cyclic cellular automaton: A system with deterministic dynamics which emulates an interacting particle system with stochastic dynamics. Journal of Theoretical Probability, 3:311-338, 1990 .

E. Formenti and P. Kůrka. Subshifts attractors in cellular automata. Nonlinearity, 20:105-117, 2007.

R. H. Gilman. Classes of cellular automata. Ergodic Theory and Dynamical Systems, 7:105-118, 1987.

P. Kůrka and A. Maass. Stability of subshifts in cellular automata. Fundamenta Informaticae, 52(1-3): $143-155,2002$.

A. Rényi. Probability Theory. Elsevier, 1970. 